Nota de investigación

\title{
Tipología de productores de uchuva en el departamento de Nariño, Colombia
}

David Rodríguez Puertas

Lizeth Tatiana Luna Mancilla

José Manuel Campo Quesada

Gissela Fernanda Guerrero Diaz

Diego Hernán Meneses Buitrago

Housseman Steven Ramos Zambrano

Luis Felipe Rincón Manrique ${ }^{\S}$

Corporación Colombiana de Investigación Agropecuaria-Agrosavia-Centro de Investigación Obonuco. Vía Pasto-Obonuco km 5, Pasto-Nariño, Colombia. (dpuertas@agrosavia.co; lluna@agrosavia.co; jcampoq@agrosavia.co; gfguerrero@agrosavia.co; dmeneses@agrosavia.co; hramos@agrosavia.co).

${ }^{\S}$ Autor para correspondencia: lfrincon@agrosavia.co

\section{Resumen}

El segmento de producción de frutas exóticas por un buen tiempo se ha venido ubicando como una importante alternativa productiva y económica ante los cultivos tradicionales en varias regiones del país. La uchuva (Physalis peruviana L.) en particular ha presentado un notable incremento en áreas productivas y comercio internacional que la consolida como una especie con alto potencial en el segmento de las frutas de clima frío. En el departamento de Nariño (Colombia) la introducción del cultivo es relativamente reciente por lo cual persiste una necesidad de conocer en detalle las condiciones productivas y ambientales en las cuales se produce el cultivar y las características socioeconómicas de los productores de la región. Para esto se diseñó una encuesta de caracterización aplicada a productores de uchuva en el departamento de Nariño entre 2019 y 2020. La información obtenida fue sometida a análisis multivariados factorial de datos mixtos (FAMD) y análisis de correspondencias múltiples MCA para variables categóricas y el análisis jerárquico tipo clúster, a través del algoritmo de Ward, como resultado se identificaron ocho variables con poder discriminatorio que conformaron tres clústeres. Cada grupo con características distintivas con relación al área de producción, grado tecnificación del cultivo, uso de mano de obra y estrategias de comercialización, entre otras, que otorgan una clara especificidad. Con la información generada se espera desarrollar estudios orientados a realizar ajustes a los modelos productivos agronómicos adaptados a las condiciones ambientales y socioeconómicas de cultivadores. Adicionalmente, los resultados obtenidos constituyen un insumo fundamental para la orientación de políticas de fortalecimiento de la cadena regional.

Palabras clave: agricultura familiar, análisis estadístico, frutas andinas, tipología.

Recibido: julio de 2021

Aceptado: agosto de 2021 


\section{Descripción del sistema y estrategias metodológicas}

Este manuscrito es parte de los resultados de investigación del proyecto 'ajuste de un modelo agronómico productivo de las variedades mejoradas de uchuva Andina y Dorada en zonas productoras de Colombia'.

En Colombia la uchuva (Physalis peruviana L.) es una de las especies frutales con importante margen de crecimiento y desarrollo con un área sembrada de 1.713 ha y rendimientos promedios de $12.4 \mathrm{t} \mathrm{ha}^{-1}$ el cultivo se ubica entre las primeras cinco frutas frescas de exportación en el país, después del banano, aguacate, plátano y gulupa (MADR, 2019). Con mercados de destino internacional como los Países Bajos, Alemania y Estados Unidos de América, además de liderar las exportaciones de la fruta a nivel de América Latina, seguido por Perú y Ecuador.

A escala nacional, Nariño ocupa el cuarto lugar con una participación de $6 \%$ de la producción total con un rendimiento de $6.8 \mathrm{t} \mathrm{ha}^{-1}$ reportado para el año 2018 (MADR, 2019), adicionalmente, viene constituyéndose en un cultivar de importancia debido a los programas de fomento, consolidación de canales de comercialización, menores afectaciones por enfermedades entre otros aspectos, que otorga un lugar relevante al cultivar a nivel regional.

La uchuva al igual que los diferentes sistemas de producción agrícola incluye múltiples componentes o subsistemas que interactúan en el tiempo y en el espacio (Coronel y Ortuño, 2005). Estos sistemas de producción o fincas no están organizados en forma idéntica, cada una tiene elementos propios y únicos que determinan propiedades y características irrepetibles. Sin embargo, muchas veces estas comparten rasgos y propiedades que las hacen semejantes en algunos aspectos y sus semejanzas permiten agruparlas para diversos propósitos (García y Calle, 1998).

Bajo esta conceptualización, el análisis de tipología agrícola brinda una posible solución, que simplifica la enorme diversidad en los sistemas complejos, construyendo clústeres (grupos) que implican la clasificación de fincas sobre las cuales se podrían hacer recomendaciones semejantes (Alvarez-Sánchez et al., 2019). Por tanto, es fundamental la caracterización y tipificación de los productores agrícolas, que coadyuve al diseño de políticas públicas que aporten a la disposición de tecnologías y apoyos gubernamentales como base de la competitividad agrícola en las regiones (González-Flores et al., 2018).

En este sentido, en el siguiente manuscrito se presentan los resultados del trabajo de investigación orientado a tipificar los productores de uchuva en el departamento de Nariño. Este estudio se realizó en dos zonas productoras de uchuva del departamento de Nariño, la primera localizada en el sur, conformada por los municipios de Ipiales, Pupiales, Puerres, Gualmatán y Córdoba y la segunda en el norte del departamento, integrada por el municipio de San Pablo. La recolección de información se elaboró a partir de una encuesta aplicada a 133 productores identificados a través de la técnica de muestreo no probabilístico bola de nieve (Ortega et al., 2017).

En el caso de la identificación de las variables para la tipificación de productores, se empleó las recomendaciones de González et al. (2018) usando en variables cuantitativas el coeficiente de variación $(\mathrm{CV})$ y en categóricas el índice de variabilidad cualitativa qualitative variability index (ICV) por sus siglas en inglés, con un criterio de selección mayor al 70\% para el primero de ellos y en variables categóricas de 0.7 (Alvarez et al., 2019). 
Una vez seleccionadas se procedió a realizar un análisis multivariado factorial de datos mixtos (FAMD) (Kassambara, 2017), acompañado de un análisis de agrupamiento jerárquico tipo clúster, empleando el método del algoritmo de Ward (Peña, 2002). Para el desarrollo de los métodos estadísticos, se usaron las librerías FactoClass (Elias et al., 2018); Factoextra (Kassambara y Mundt, 2020); FactoMiner (Husson et al., 2020); dplyr (Wickham et al, 2019) del software R v.3.2.2 ${ }^{\circledR}$ (R DevelopmentCore Team, 2013). Para validar esta tipificación de productores, se realizó taller con un grupo de productores y técnicos expertos del sistema productivo uchuva.

\section{Tipología de productores de uchuva}

Como resultado de la aplicación de la encuesta de caracterización productiva y socioeconómica aplicada a los productores de uchuva se recolectaron ciento sesenta y ocho (168) variables cuantitativas y cualitativas las cuales cumplieron con los criterios anteriormente mencionados, de las cuales se seleccionaron veintiséis (26). Estas variables fueron sometidas a la aplicación de análisis ACP, lo que permitió realizar una segunda depuración, para descartar las variables que presentaban menor significancia en las dimensiones. Como resultado se seleccionaron ocho variables (cuatro cualitativas y cuatro cuantitativas).

Que fueron las más determinantes para el sistema productivo: experiencia en el cultivo (CV: 100.9\%), área total (CV:190.7\%), total de plantas en producción (CV:182.4\%). alternativas de producción (CV:66.1). Aunque la variable descrita no cumple con el criterio de CV (superior al $70 \%$ ), se incluyó en el análisis jerárquico debido a su importancia en el sistema productivo para la continuidad del cultivo.

Asistencia técnica (IVC: 0.99), tipo de mano de obra (IVC: 0.96), certificación del lote (IVC: 0.81), nivel de tecnificación (IVC: 0.83). Como resultado del análisis estadístico, se logró reducir la información en cuatro dimensiones que concentran $67.7 \%$ de la varianza total, las dimensiones uno y dos acumulan 47.4\% de la varianza. Autores como Alvarez et al. (2014) afirman que una varianza superior de $60 \%$ es significativa en los análisis multivariados.

Las variables con mayor aporte a la dimensión uno fueron: total plantas en producción (22.9\%), área real en uchuva (19.09\%), experiencia en la producción de uchuva (15.61\%), nivel de tecnificación (14.49\%) y alternativas comerciales (13.04\%), en resumen, la dimensión uno, aporta información de los diferentes componentes del estudio (técnico, social y económico), por su parte, para la dimensión dos, las variables que más aportaron a la varianza se encontraron; asistencia técnica $(27.87 \%)$, certificación de la finca $(26.2 \%)$ y nivel de tecnificación $(21.95 \%)$, esta dimensión, da información acerca del componente técnico del estudio. Finalmente, en las dimensiones tres y cuatro, las variables con mayor significancia fueron mano de obra dominante en el cultivo (contratada y familiar) $(72.93 \%, 47.02 \%)$ y nivel de tecnificación del cultivo $(24.98 \%$, $36.38 \%)$.

Para el desarrollo de este estudio, se obtuvieron tres clúster o tipos de productores, diferenciados a una distancia euclidiana de 0.64 como se puede observar en mapa de factores (Figura 1). Donde el clúster número uno, agrupó $54.13 \%$ de la muestra (72 productores), el número dos el $36.09 \%$ de la muestra (48 productores) y el número tres $9.77 \%$ (13 productores). Una vez identificados los clústeres, se validó la información obtenida en campo a través de la socialización con expertos locales en el sistema productivo. 


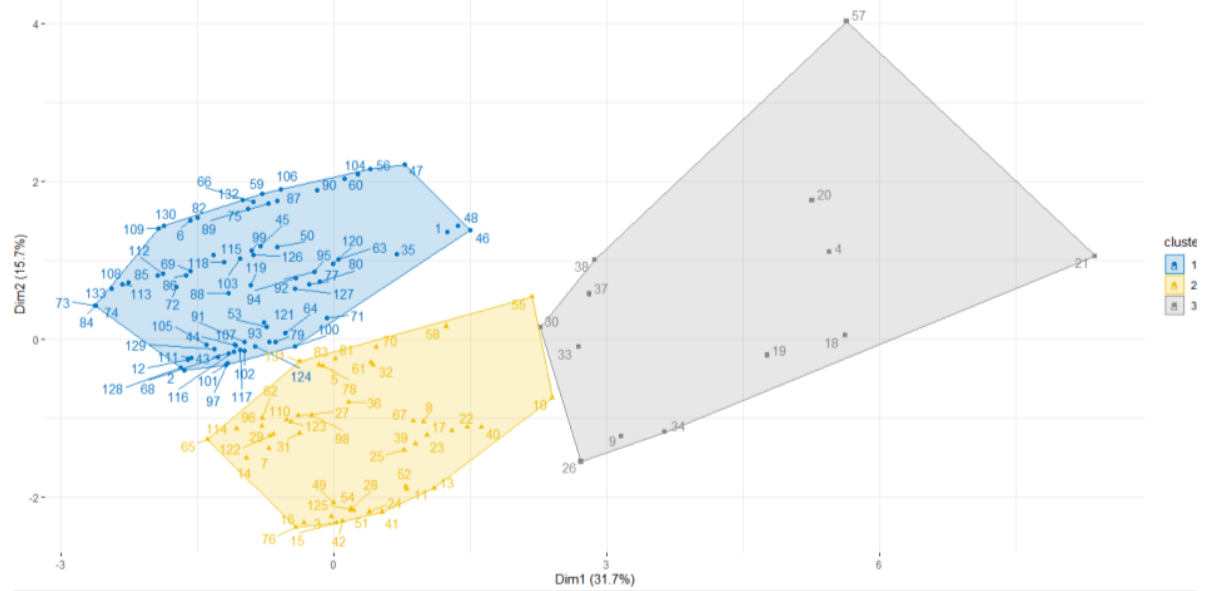

Figura 1. Mapa de factores por individuos según agrupación de clúster para la tipificación de productores de uchuva del departamento de Nariño.

Clúster 1. Pequeños productores con bajo nivel de tecnificación y baja experiencia en el cultivo

Este grupo está compuesto por $54.1 \%$ de los casos estudiados, está explicado principalmente por las variables: certificación de lote, nivel de tecnificación y prestación de asistencia técnica. Se conforma por productores que poseen en promedio de 0.35 ha sembradas en uchuva, con extensiones máximas de 1.8 ha y tienen en promedio 302 plantas en producción, también se encuentran productores con cultivos en estado de renovación y cultivos que aún no inician su etapa productiva.

Se caracterizan por tener una baja experiencia, en promedio 2.98 años, su nivel de tecnificación es bajo, dado que ninguno de los lotes cuenta con certificaciones de calidad, la mayoría no realizan análisis de suelos, no aplican enmiendas, no cuentan con sistema de riego y no reciben asistencia técnica por parte de personal calificado. En el aspecto comercial, cuentan con pocas ofertas para vender su producción, en promedio cuentan con 1.25 alternativas comerciales y la presentación de venta predominante es la uchuva sin capacho. Principalmente están ubicados en la zona norte del departamento y gran parte de los ingresos familiares se derivan únicamente de la producción de la uchuva, la mayoría se encuentra en condición de pobreza, según resultados de NBI.

\section{Clúster 2. Pequeños productores, con nivel medio de tecnificación y con diversificación de cultivos}

Compuesto por $36.1 \%$ de los casos estudiados, se explica principalmente por las variables: Nivel de tecnificación, asistencia técnica y certificación de lote. Se conforma por productores que poseen en promedio 0.48 ha, con un promedio de 588 plantas en producción y con una experiencia media de 3.37 años. Gran parte se ubica en la zona sur del departamento, cuenta con servicio de asistencia técnica, lotes certificados y nivel de tecnificación media. En general estos productores llevan registros, no usan créditos para el cultivo de uchuva y tienen el hábito del ahorro. La presentación de uchuva con capacho es la predominante para la comercialización y los ingresos familiares se derivan de la venta de la uchuva y otros productos agropecuarios. 


\section{Clúster 3. Medianos productores con mayor experiencia y nivel de tecnificación alto}

Está compuesto por $9.8 \%$ de los casos estudiados, las variables que explican en mayor medida a este grupo son: plantas en producción, área en uchuva y años de experiencia. Está conformado por los productores con mayor experiencia en el cultivo (11.23 años), en comparación con los otros grupos, representa en promedio el mayor número de plantas en producción (3.8937) y de área sembrada (3.34 ha). La mayoría cuenta con asistencia técnica, certificaciones de calidad, riego, análisis de suelo y aplicación de enmiendas, por lo cual su nivel de tecnificación es alto. En el aspecto comercial, cuentan con 2.92 alternativas comerciales, y manejan dos presentaciones para la venta: uchuva con y sin capacho. Los productores llevan registros, tienen el hábito de ahorro y apalancan su cultivo de uchuva mediante créditos.

\section{Conclusiones}

El proceso de validación presentó coherencia entre la conformación de los grupos de productores y su asociación con la realidad del territorio y el sistema productivo, a partir de los criterios sociodemográficos, técnicos, económicos y organizacionales. La tipificación determinó tres grupos de productores claramente diferenciados entre los cuales se contienen las principales características técnicas, productivas y de comercialización de los cultivadores de uchuva en el departamento de Nariño.

El agrupamiento por segmentos arroja entre el grupo 1 y 2 la ausencia de diferencia significativa en las variables: área en uchuva, plantas en producción, años de experiencia y alternativas comerciales; siendo la ausencia de asistencia técnica, un bajo nivel de tecnificación y carencia de certificaciones de lotes, condiciones determinantes para ubicar el primer grupo en un bajo nivel de desarrollo técnico y comercial. En tanto, para el segundo segmento de productores contar con asesoría técnica, un nivel medio de tecnificación de la producción y certificaciones de lotes los ubica en un nivel medio de tecnificación del sistema productivo. Para el tercer segmento de productores las variables área en uchuva, plantas en producción, experiencia en el cultivo y alternativas comerciales reflejan una marcada diferencia en comparación con los grupos 1 y 2.

El tiempo de experiencia en el manejo del cultivo es un factor determinante debido al relativo poco tiempo que tiene la introducción de la uchuva en el departamento, esto reflejado en el tiempo promedio de experiencia que ostentan los productores. Lo que lleva a que quienes tienen más tiempo de producción pueden tener un mejor conocimiento del manejo agronómico del cultivo logrando mejores prácticas culturales orientadas a un proceso de tecnificación gradual de la producción. Esto se apalanca con el acceso a servicios de asistencia técnica, ya sea contratada, servicios públicos o prestados por comercializadores, que permiten realizar un acompañamiento a los productores para incorporar gradualmente adecuadas prácticas productivas.

La certificación de lotes que garantiza a los productores la posibilidad de acceder a mercados diferenciados y por tanto diversificar sus alternativas comerciales responde al proceso continuo de tecnificación del cultivo adelantado por un segmento de éstos. El estudio concluyó el amplio margen de crecimiento que tiene el cultivo en el departamento de modo que pueda constituirse en alternativa para la diversificación de ingresos de los productores para enfrentar fluctuaciones de los precios de los cultivos tradicionales. 


\section{Agradecimientos}

Los autores agradecen el financiado de la Corporación Colombiana de Investigación Agropecuaria (Agrosavia), ID: 1000642a. A Pedro Rodríguez Hernández, Carlos Andrés Benavides, Andrea Castro Jiménez y Martín José Jairo Piscal por sus contribuciones en diferentes etapas del desarrollo de este trabajo.

\section{Literatura citada}

Alvarez-Sánchez, D. E.; Gómez-López, E. D. y Ordóñez-Hurtado, H. R. 2019. Tipología de fincas productoras de arveja (Pisum sativum L.) en la subregión sur de Nariño, Colombia. Ciencia y Tecnología Agropecuaria. 20(3):659-67. https://doi.org/10.21930/rcta.vol20_num3_ art:1593.

Alvarez, S.; Paas, W.; Descheemaeker, K.; Tittonell, P. y Groot, J. 2014. Construcción de tipologías, una forma de manejar la diversidad de las fincas: directrices generales para humidtropics. $40 \mathrm{p}$.

Coronel-Renolfi, M. y Orduño, P. S. F. 2005. Tipificación de los sistemas productivos agropecuarios en el área de riego de santiago del estero, Argentina. Problemas del Desarrollo. Rev. Latinoam. Econ. 36(140):63-88. https://doi.org/10.22201/iiec.20078951e. 2005.140.7572.

Elias, C.; Campo, P.; Torres, C.; Díaz, I.; Sadinle, M. y Medina, J. 2018. Package 'factoextra'. https://cran.r-project.org/web/packages/FactoClass/FactoClass.pdf. 1-34 pp.

García, C. H. y Calle, L. M. 1998. Consideraciones metodológicas para la tipificación de sistemas de producción bovina a partir de fuentes secundarias. Ciencia y Tecnología Agropecuaria. 2(2):6-15. doi: 10.21930/rcta.vol2_num2_art:166.

González-Flores, S.; Guajardo-Hernández, L. G.; Almeraya-Quintero, S. X.; Pérez-Hernández, L. M. y Sangerman-Jarquín, D. M. 2018. Tipología de productores de maíz en los municipios de Villaflores y La Trinitaria. Chiapas. Rev. Mex. Cienc. Agríc. 9(8):1763-1776. https://doi.org/10.29312/remexca.v9i8.1722.

Husson, F.; Josse, J.; Le, S. and Mazet, J. 2020. FactoMineR: multivariate exploratory data analysis and data mining. https://cran.r-project.org/web/packages/FactoMineR/index. html. 25(1):1-18.

Kassambara, A. 2017. Practical guide to principal component methods in R, PCA, (M) CA, FAMD, MFA, HCPC, factoextra. Statistical tools for high-throughput data análisis (STHDA). 1 ed. Francia. 29 p.

Kassambara, A. and Mundt, F. 2020. Factoextra: extract and visualize the results of multivariate data analyses. R. package version 1.0.7. https://cran.r-project.org/web/packages/factoextra /factoextra.pdf.

MADR (Ministerio de Agricultura y Desarrollo Rural). 2019. Dirección de cadenas agrícolas y forestales. Cadena de la uchuva. Colombia. $18 \mathrm{p}$.

Ortega, R.; Sonego, M.; Pulido, J.; Crespo, A.; Mejías, E. y Sordo, L. 2017. Métodos indirectos para la estimación de poblaciones ocultas. Rev. Españ. Salud. Públ. 91:1-9. https://www.redalyc.org/articulo.oa?id=17049838040.

Peña, D. 2002. Análisis de datos multivariantes. McGraw-Hill. $1^{\text {ra. }}$ ed. España. 201-226 pp.

Wickham, H.; Romain, F.; Lionel, H. y Kirill, M. 2019. Dplyr: a grammar of data manipulation. R. package version 1.0.0. 